\title{
Pathology of the intima in coarctation of the aorta: a study using light and scanning electron microscopy
}

\author{
ALEXANDER KENNEDY, D G TAYLOR, AND T E DURRANT \\ From the Departments of Pathology and Cardiothoracic Surgery, Northern General Hospital \\ and University of Sheffield Medical School, Sheffield, UK
}

ABSTRACT In the adult type of coarctation of the aorta the lumen is constricted by a shelf of fibromuscular tissue that protrudes from the dorsal wall of the aorta at a point near the site of insertion of the ligamentum arteriosum. The intima covering this constricting shelf has been studied using conventional light microscopy and scanning electron microscopy of surgical specimens of coarctation removed at ages varying from 2 to 46 years. Immediately proximal to the stenosis, the intima was found to run in fine longitudinal folds that radiated into the orifice. On the distal side of the shelf the intima was grossly irregular with deep longitudinal and transverse folds that gave it a convoluted appearance resembling the surface of the brain. In adults and older children the intima immediately distal to the orifice was covered with a laminated layer, distinct from the elastica, which often gave positive staining reactions for fibrin. Intimal thickness increased irregularly with age, but large increases of $500 \mu \mathrm{m}$ or greater were found only in patients over the age of 15 years. These findings are consistent with the view that the constriction has both a fibroelastic component that is congenital and a fibrous component that is acquired and progressive. It is suggested that this progressive element is caused by deposition of fibrin on an abnormal intimal surface in a region of turbulent blood flow. Such a mechanism would explain the occasional recurrence of coarctation of the aorta after surgery. This may be due to failure to remove the abnormal area of endothelium completely and to persistent abnormalities of blood flow at the site of anastomosis.

Coarctation of the aorta is one of the few truly curable causes of hypertension and, as such, it is important despite its being an infrequent condition. Apart from the production of systemic hypertension coarctation is associated with several secondary effects on the vascular system both in the formation of a collateral circulation and in modification of the aorta itself. Clagett and his colleagues (1954) pointed out that the severity of the stenosis cannot be judged from the external appearance of the aorta, and that intimal changes seen in adult cases of coarctation may cause technical problems at operation. It is well established that the primary defect is "a curtain-like infolding of the media that involves the superior, anterior, and posterior aspects of the aorta. The lower portion of the aortic wall, or that portion into which the ductus arteriosus inserts, does not take part in the deformity" (Clagett et al, 1954). While some authors have concentrated their attentions on this medial deformity (Wielenga and
Dankmeijer, 1968; Hutchins, 1971; Talner and흥 Berman, 1975) much of the stenosis may be due to an intimal component (Edwards et al, 1948; Clagett et al, 1954) that in some cases causes complete occlusion. These authors also presented evi-응 dence that the intimal component is not present at birth and is seen only in lesions of some dur-음 ation. We have studied this progressive intimal $\stackrel{N}{\sim}$ element in a series of surgically removed coarcta- $\sigma$ tions using both conventional light microscopy and $N$ scanning electron microscopy.

\section{Materials and methods}

The material consisted of 27 coarctations mainly resected by DGT and collected prospectively. The 0 patients (20 male and seven female) varied in age ${ }_{0}^{\circ}$ from 14 months to 46 years. All had been referred $\vec{\Phi}$ to the regional cardiothoracic unit, which draws patients from South Yorkshire, Nottinghamshire, 응 Derbyshire, and Lincolnshire. 
The proximal end of each specimen was identified usually by means of a suture and was washed in a vigorous stream of isotonic saline before fixation. All were described and measured by one of us using a stereomicroscope.

After fixation in formal saline for at least 48 hours most of the specimens were divided longitudinally through the orifice, if present. The plane of section was dorsoventral so that it ran through both the orifice and the ligamentum arteriosum. One half was embedded in paraffin, and conventional paraffin sections were prepared to give views of the type shown in fig 1 . These were stained with haematoxylin and eosin $(\mathrm{H}$ and E), Verhoeff's elastic stain, and van Gieson (EVG), Martius scarlet blue (MSB), phosphotungstic acid haematoxylin (PTAH), and by Mowry's alcian blue periodic acid Schiff technique (AB-PAS). Measurements were made using an eye piece graticule.

The opposite half of the specimen, and in some cases the whole specimen, was used for scanning electron microscopy. After dehydration in acetone and critical point drying the specimens were fixed to aluminium stubs with Dag 915 conductivity paint, coated with gold, and examined on a Cam-

bridge S-4 Stereoscan and a Philips SEM 501 scanning electron microscope using accelerating voltage of $10 \mathrm{kv}$ and $15 \mathrm{kv}$.

\section{Results}

Using the light microscope the findings were similar to those described by Edwards et al (1948) and by Clagett et al (1954). The lumen was constricted eccentrically by a shelf of tissue protruding from the dorsal and lateral walls of the aorta (fig 1). This shelf had two components, one of which was composed of fibroelastic tissue and was constantly present. The other component was a variable degree of intimal thickening that in some cases was a much greater factor in constricting the lumen than the medial deformity. In three cases the lumen was completely occluded.

While the elastic component tended to point towards the site of insertion of the ductus, as described by Wielenga and Dankmeijer (1968), the intimal element was most prominent in the downstream side of the orifice and was directed not towards the ductus but in the direction of blood flow.

This intimal thickening was composed of lamin-

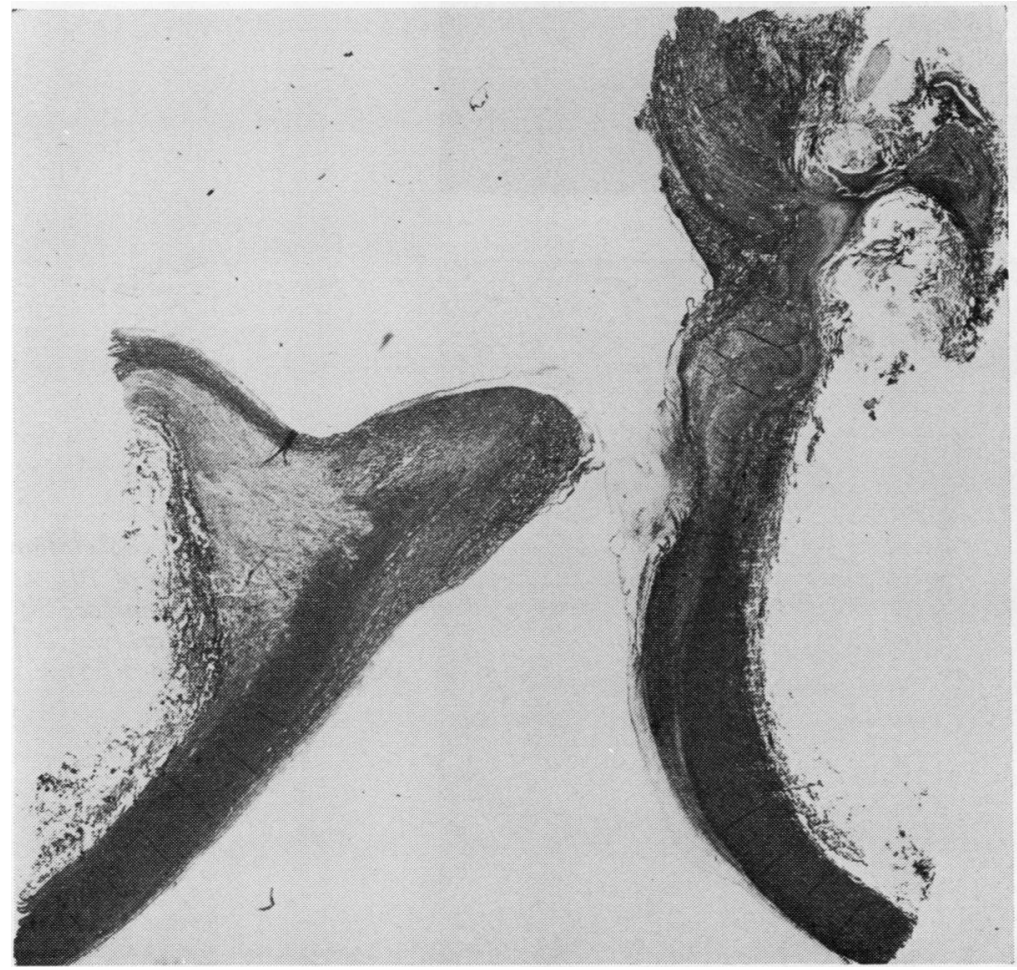

Fig 1 Longitudinal section through orifice of a coarctation resected from man of 22. In top right hand corner ligamentum arteriosum is crushed by ligature and shelf arising from dorsal wall points towards this area. Faint outline of thickened intima can be seen on edge of shelf and on ventral wall below (distal to) orifice $(E V G \times 10)$. 
ations of collagen and elastic fibres lying parallel with the media (fig 2). Cells resembling smooth muscle cells were present, and in 13 cases there was definite evidence of the presence of fibrin as shown by the MSB and PTAH techniques (fig. 3). In the examples of more gross thickening, orientation of the laminations appeared to alter progressively from deeper to more superficial layers. Assuming that this was a progressive lesion, it was possible in some cases to see not only a gradual diminution of the lumen with age but also to infer a gradual alteration in the axis of blood flow $\vec{F}$ through the orifice (fig 4).

The depth of the intimal thickening was irregu- $\frac{}{-}$ larly related to the age at the time of operation (see table), being least in childhood and greatest $\vec{\nabla}$ in those over 25 years of age. No patient below $\propto$ the age of 15 had intimal thickening to a depth is of more than $500 \mu$. The differences between the $\vec{\circ}$ mean depth of the plaque in older subjects, as compared with those in children, were statistically $\vec{\omega}$ significant. There was no obvious tendency for

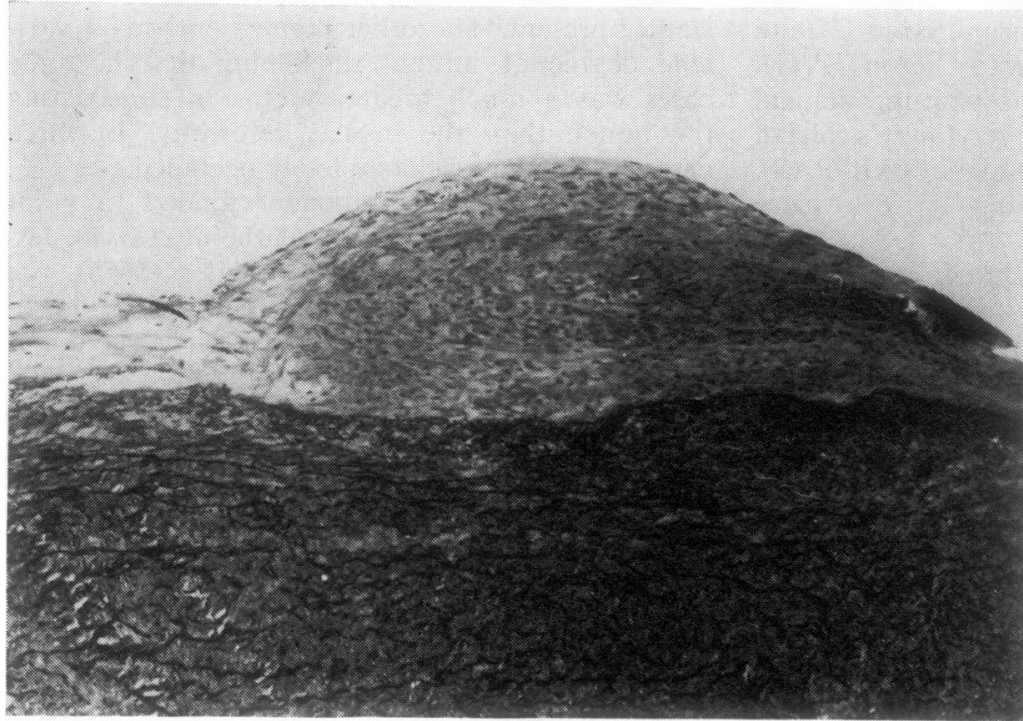

Fig 2 Thickening of intima in man of 18. This was situated on distal side of shelf in dorsal wall. Laminated intimal thickening stands out in contrast to dark media. Lesions of this sort were not found in children (PTAH $\times 25)$.

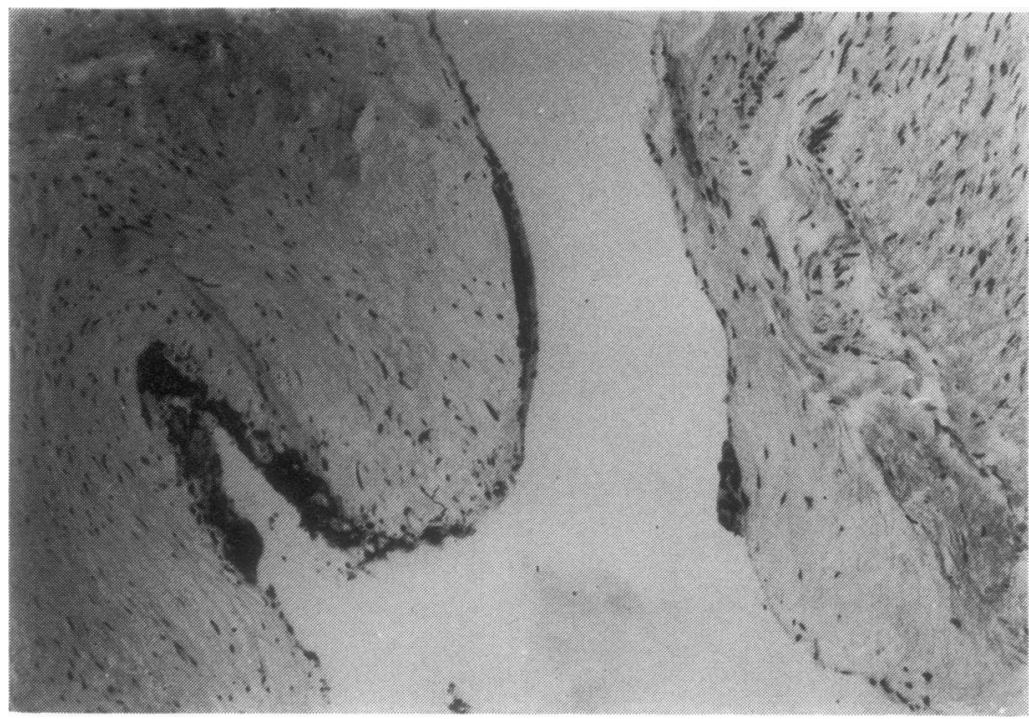

Fig 3 Orifice of a coarctation removed from man of 20 . Thickened intimal surface is encrusted with darkly stained fibrin $(M S B \times 75)$. 


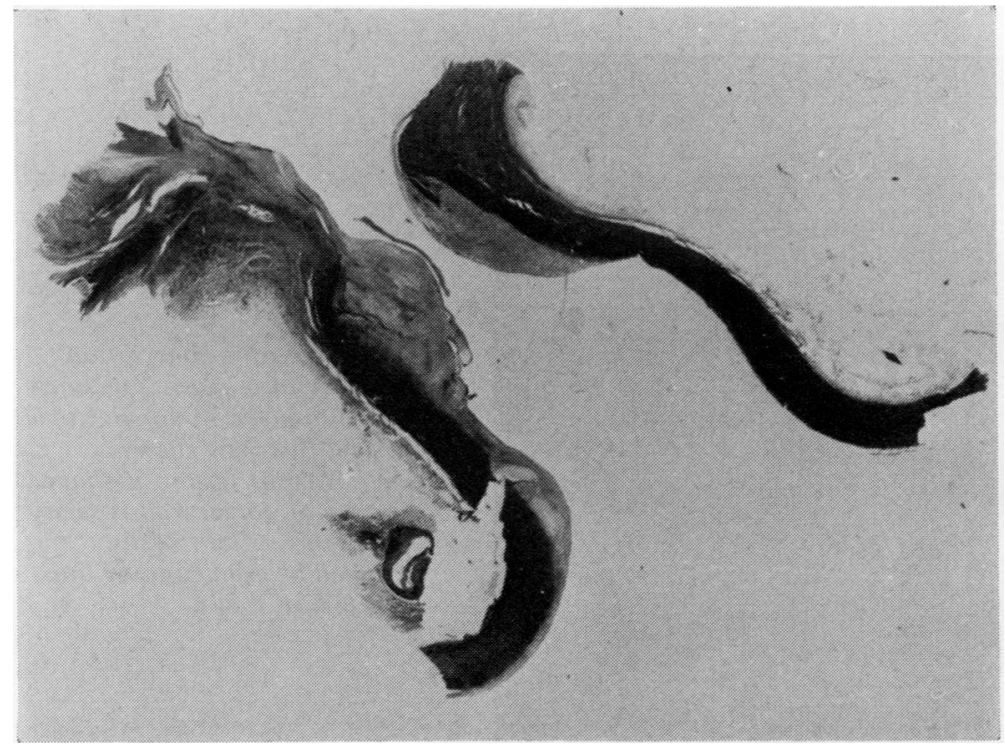

Fig 4 Woman aged 32 at time of operation. Proximal part of aortic lumen is on left with ventral wall lowermost. Dorsal shelf in media is only modest in size but distal to this obstruction there is enormous degree of intimal thickening, so much so that, in this case, the intimal component caused more stenosis than the medial deformity. It can also be seen that in early life stream of blood would have been directed against ventral wall but narrower part of the lumen has moved downstream and rotated dorsally $(E V G \times 4 \cdot 5)$.

Relationship between thickness of intimal component of stenosis with age at operation. Differences between thickness in children and both 25-30 group and over-30 group are significant $(\mathrm{P}<0.01)$

\begin{tabular}{llll}
\hline $\begin{array}{l}\text { Age } \\
\text { (years) }\end{array}$ & No of cases & $\begin{array}{l}\text { Mean thickness } \\
(\mu) \text { of } \\
\text { intimal component }\end{array}$ & Range \\
\hline Under 15 & 5 & 290 & $(180-400)$ \\
$15-24$ & 6 & 760 & $(240-1500)$ \\
$25-30$ & 4 & 1785 & $(540-3900)$ \\
Over 30 & 3 & 1250 & $(1000-1400)$ \\
\hline
\end{tabular}

the thickness to be greater in male than in female patients but accurate measurements were only possible in five females and they were operated on at a higher average age.

\section{SCANNING ELECTRON MICROSCOPY}

Proximal to the shelf the intima had a radial arrangement of fine folds that ran into the orifice (figs 5 and 6). In the younger cases these folds could be traced right into the orifice itself where they were often obscured by what appeared to be a fresh deposit of fibrin and red blood cells (fig 8).

Distal to the shelf the appearances were variable, but the folding of the intima was much coarser than on the proximal side and the intimal folds instead of being radially arranged were completely irregular (fig 7). In many cases a clear transition was visible at the level of the stenosis (fig 8).

In older patients the intimal pattern was often obscured at the level of the dorsal shelf by a featureless plaque. This was best developed on the downstream side of the orifice (fig 9) and it covered less of the intima on the proximal side (fig 10). This lesion which corresponds to the laminated intimal thickening seen by light microscopy was not seen in infancy, when the intima was visible throughout the length of the specimen (figs 1 and 11). In adults there were often irregular excrescences on the distal side of the constriction that gave the intima a very irregular appearance, visible under the dissecting microscope. These gave rise to irregular areas of intimal thickening as seen in paraffin sections. In one case, a 2-year-old child, two large longitudinal ridges raised and distorted the distal part of the dorsal wall of the aorta (fig 11).

\section{Discussion}

The light microscopic findings amplify and confirm the observations of Edwards et al (1948) and Claggett et al (1954). The intimal component that they describe is minimal in infancy; it increases in thickness with age, and it may lead to complete occlusion of the lumen. It may be concluded that coarctation of the aorta has a medial component that is congenital and an intimal component that is progressive so that coarctation is not a static abnormality but one that becomes worse with increasing age. Not only does the orifice become narrower but, in some cases, the appearances suggest that the axis of blood flow may alter as inti- 


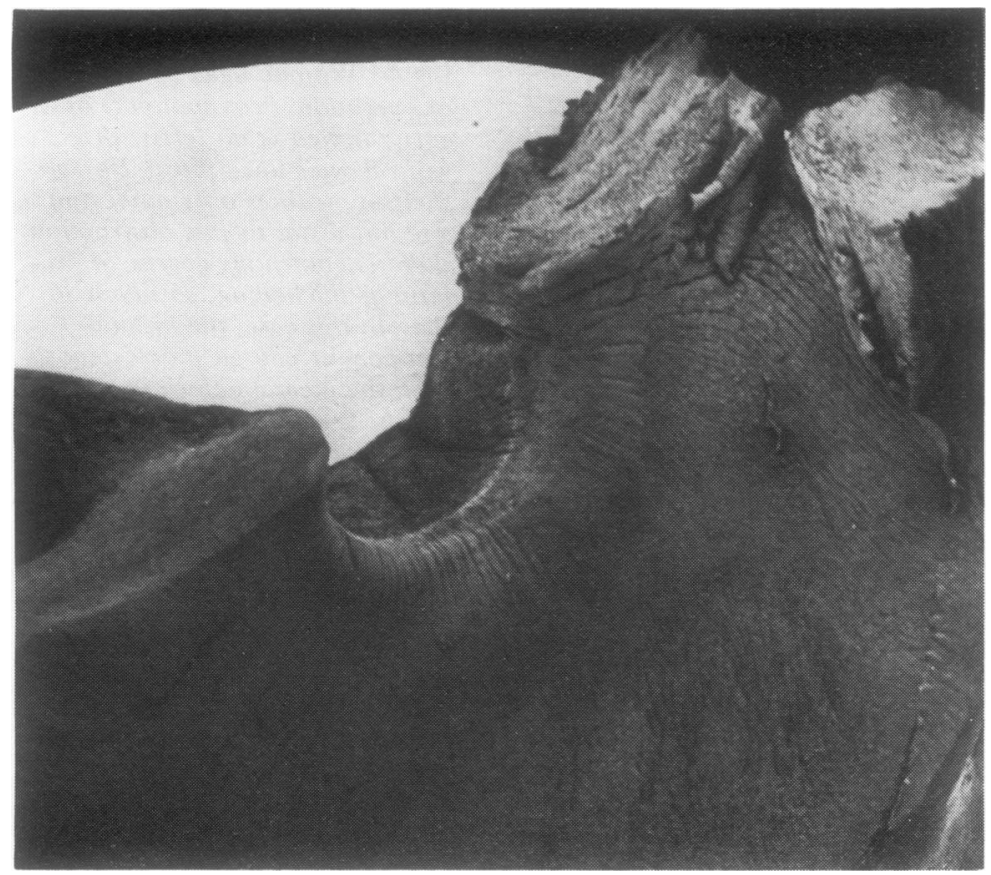

Fig 5 View of a coarctation from a boy of 6 years. Specimen has been bisected in dorsoventral plane and this view shows cephalic side of lesion. V-shaped deformity of dorsal wall is seen on left. Fine radial folds in intima can be seen running into orifice $(S E M \times 21)$.

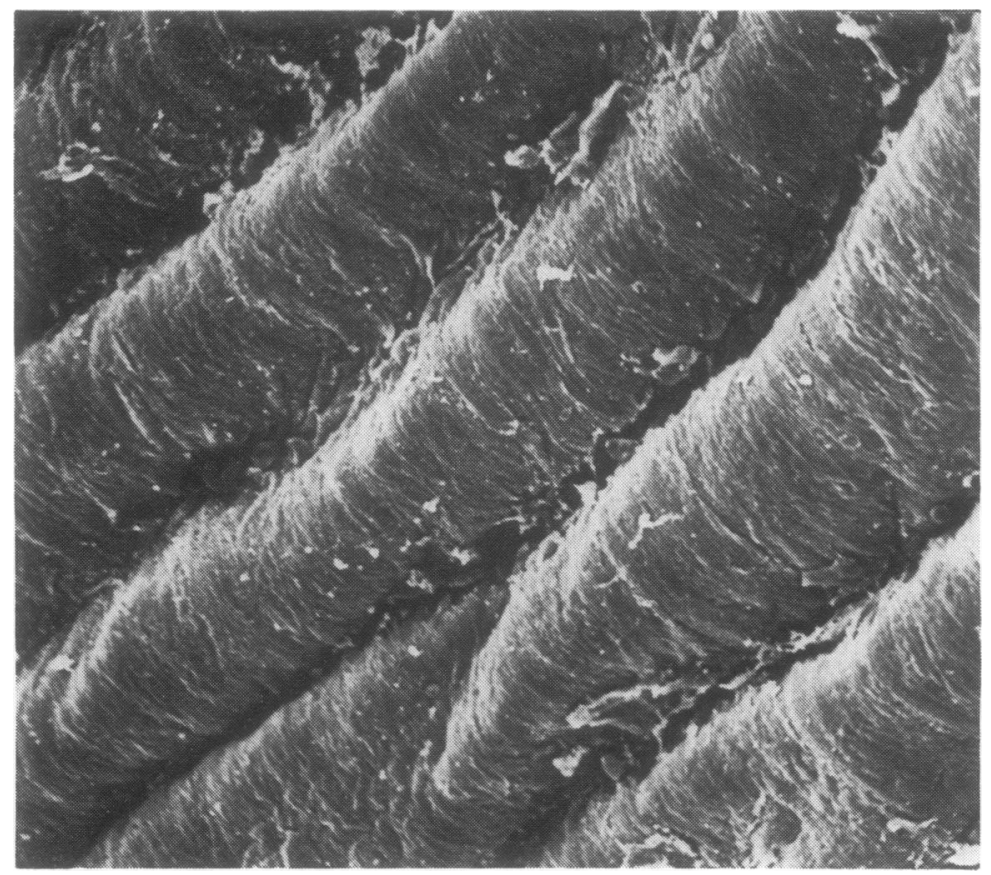

Fig 6 Radial intimal folds from cephalic side of a coarctation from a boy of 15. Surface is arranged in regular even furrows in which a few red cells and a little fibrin are adherent $(S E M \times 500)$. 


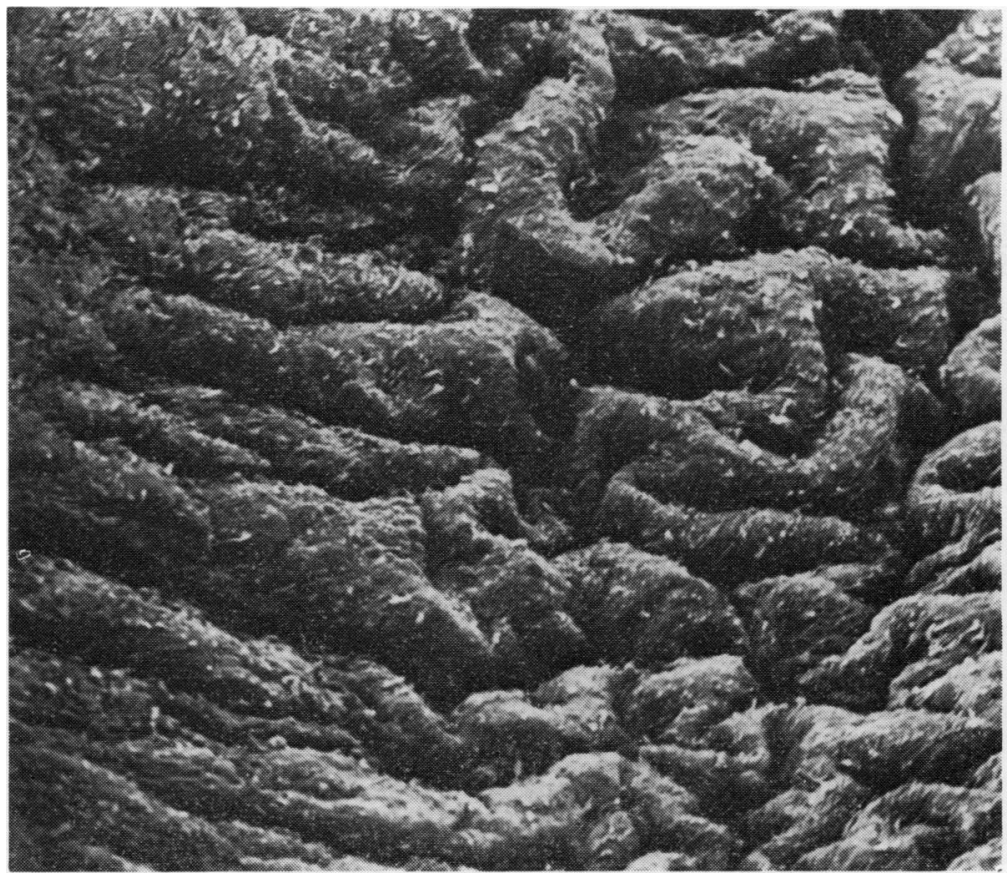

Fig 7 Boy age 15. Intima caudal to obstruction is irregularly folded and has a convoluted pattern resembling that of cerebral cortex.

Compare this with fig 6 which is from same specimen $(S E M \times 220)$.

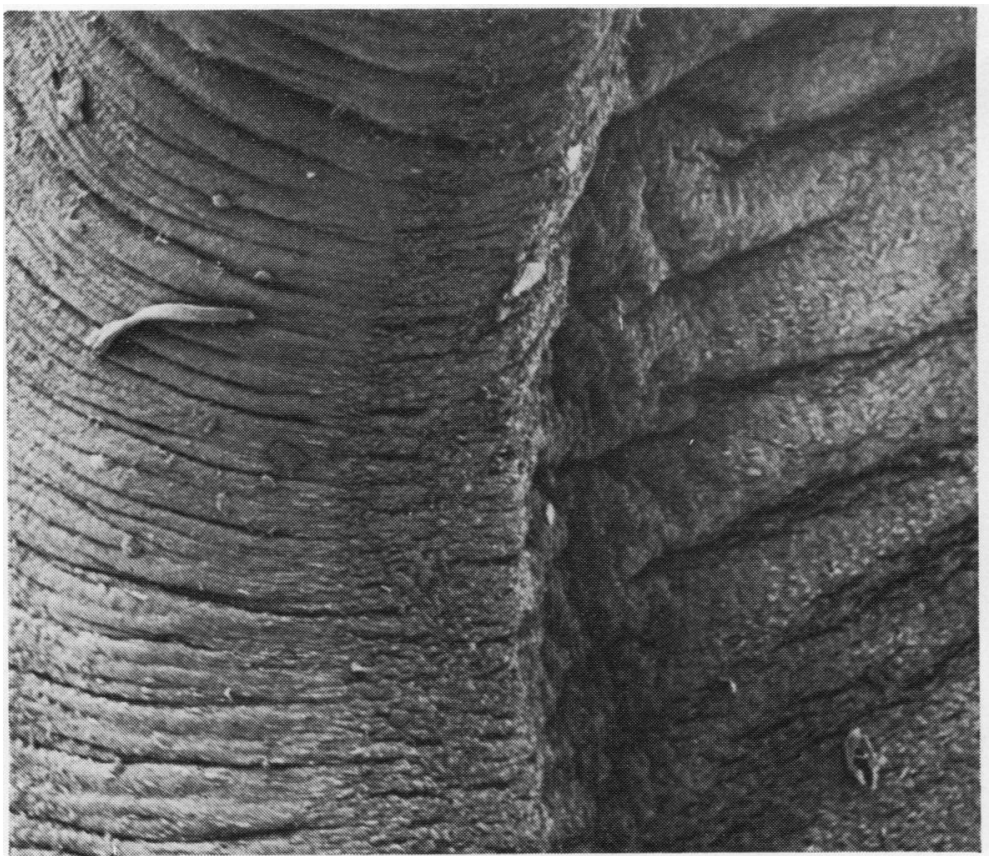

Fig 8 Lip of orifice from boy of 6 years (same case as fig 5). Cephalic aspect is on left and caudal on right. Fine intimal furrowing of cephalic aspect is shown in contrast to much coarser and irregular folds on caudal side. Red blood cells and fibrin adhere in a dense band on lip of orifice $(S E M \times 110)$. 


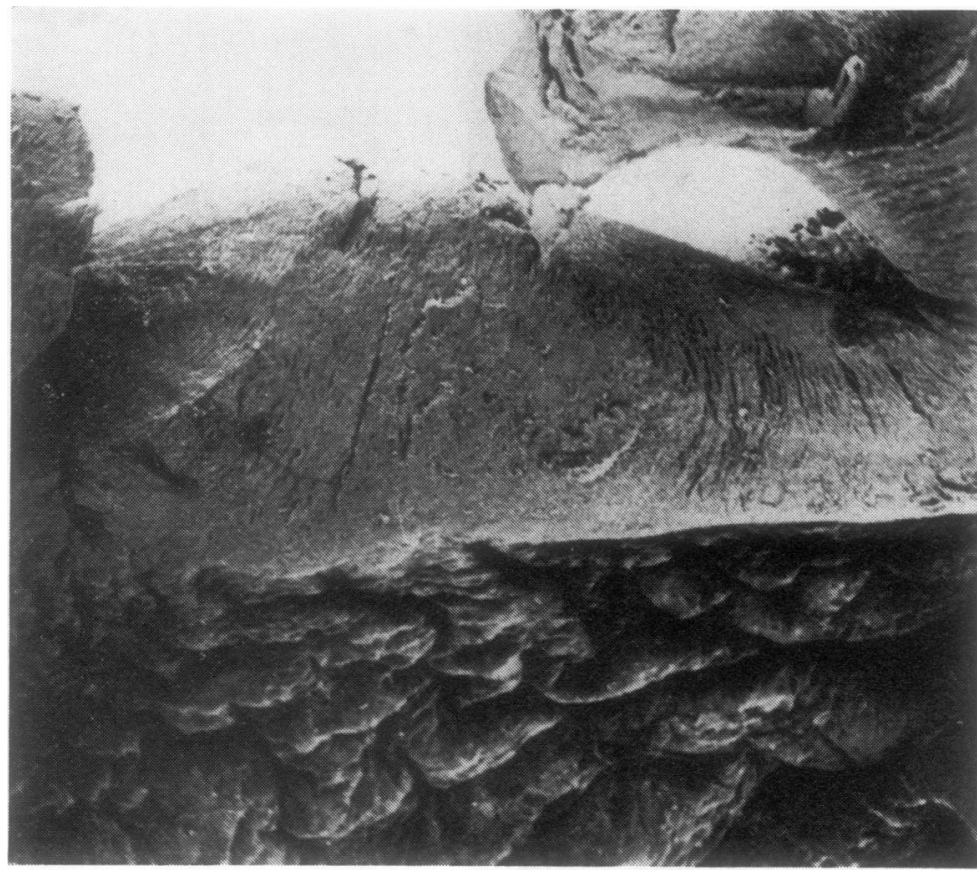

Fig 9 Distal side of obstruction in boy of 15 (same case as figs 6 and 8). Convoluted pattern of intima is seen in lower half of field but near orifice itself intima is totally obscured by featureless plaque $(S E M \times 55)$.

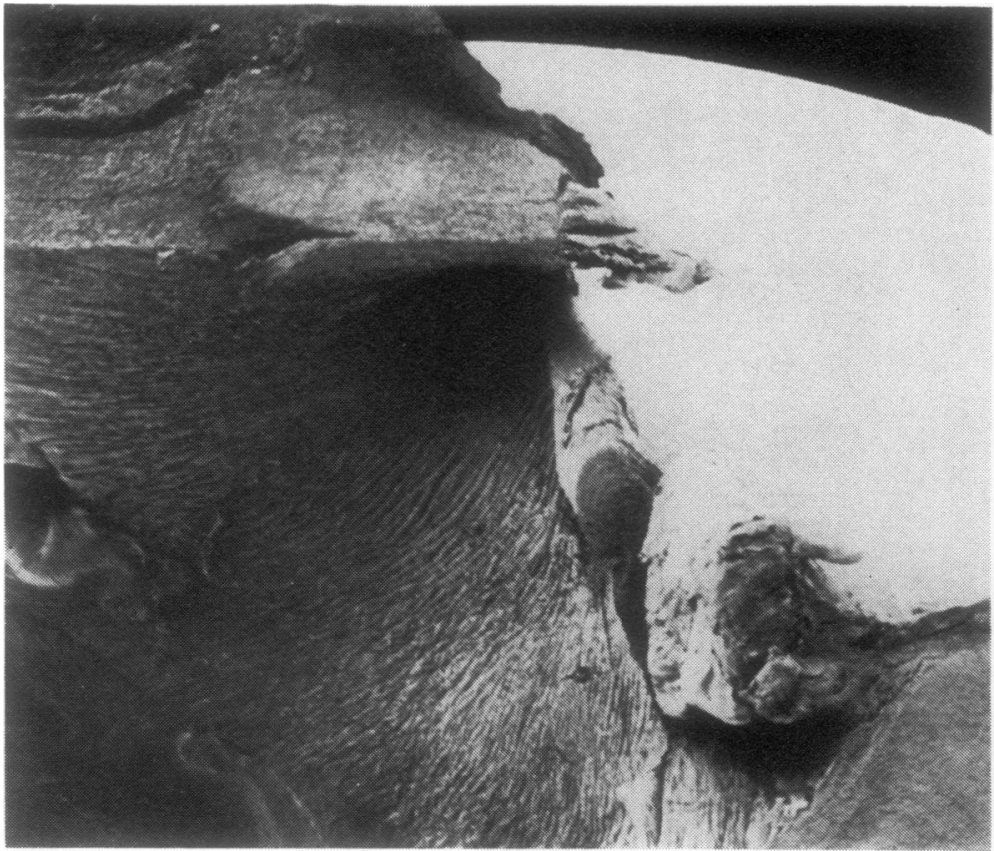

Fig 10 An oblique view of intimal plaque shown in fig 9. Fine furrows on cephalic side of obstruction disappear before they reach lip of shelf. Lip itself is covered with a plaque, cut surface of which can be seen just below and to right of centre. Laminations of this plaque are just visible $(S E M \times 21)$. 


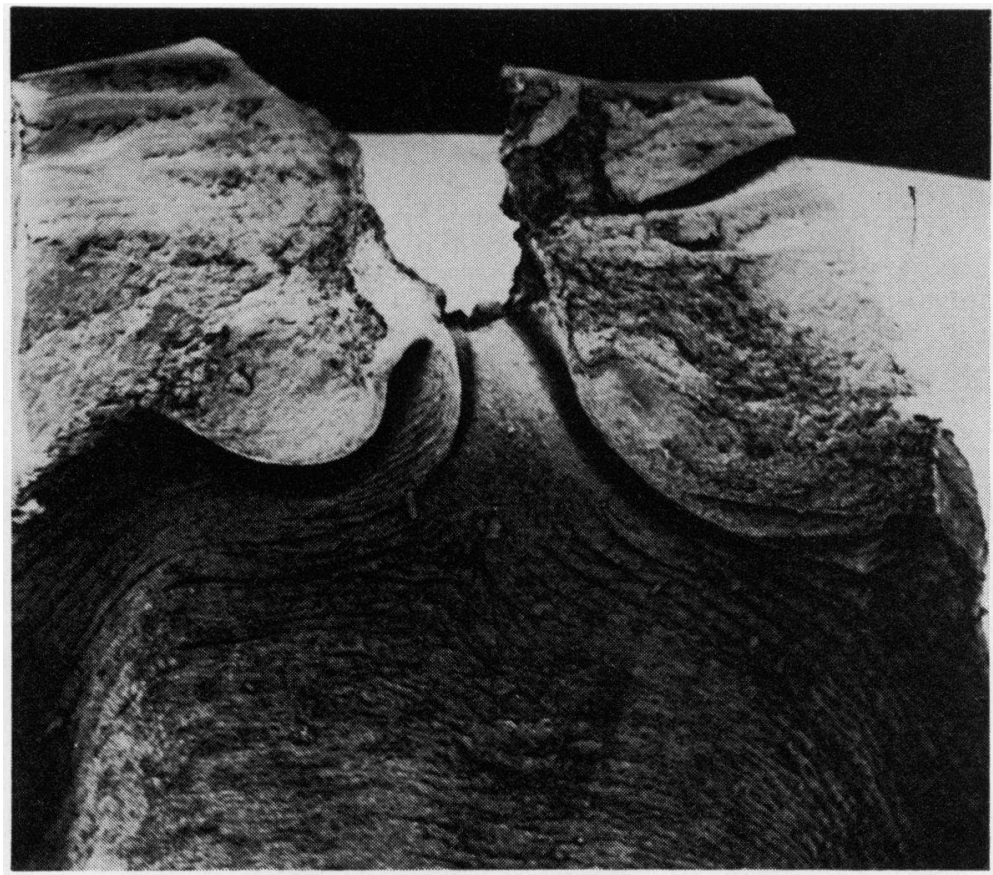

Fig 11 A longitudinal view from caudal aspect of a coarctation from a boy aged 3 years. Ventral wall has been cut away and irregular folding of dorsal intima is well seen below obstruction. Intima is visible right through to cephalic side of specimen and no fibrous plaque has formed $(S E M \times 55)$.

mal thickening builds up and deflects the jet of blood. This may lead to shifting of the sites of "jet lesions" in the distal intima.

The intimal component is laminated and often contains areas that stain for fibrin, suggesting that the layer is gradually built up by apposition of fibrin and platelets. This mainly takes place immediately downstream to the constriction, and it may be significant that adherent fibrin and blood cells were commonly seen at this site when using the scanning electron microscope. Use of this instrument confirms this view, for in older patients the intima was obscured at this site and on the cut surface the laminations of this plaque were visible (fig 9). The fibrin and platelets adhere at this site due to the turbulent conditions of flow that must occur as the stream of blood issues from the orifice. The production of eddies at the sides of the jet seems probable so that platelets from slowly moving blood may settle on the intima on the distal side of the orifice.

Our findings indicate that this intima is probably abnormal and certainly even in the very young, the distal intima is different to that on the proximal side. The significance of these ridges is still uncertain but they probably reflect the orientation of the underlying elastic fibres. Clark and Glagov (1976) examined animal material using fixation at pressures exceeding the diastolic blood pressure and found that under such conditions most of the ridges described in vascular intima were not found. Our material was fixed without pressure, and probably the fine longitudinal furrowing seen on the proximal aspect may be absent in vivo at aortic pressures. The effect of pressure on the distal convolutions at the intima seems less certain for these are coarser and irregularly arranged. Furthermore, the pressures on the distal side of the stenosis are lower than on the proximal side. Many of these distal deformities are probably present before operation, and there seems no doubt that the distal intima is abnormal compared to that on the proximal side. While the extent of intimal furrowing in vivo may be a matter of some conjecture, we have now shown that there is progressive obliteration of the intimal pattern by laminated plaque.

The existence of an abnormal area of intima in the region of a coarctation provides a possible explanation for the recurrence of this lesion. In some cases, in which the first operation is carried out in early childhood, recurrence may arise from a relative failure of growth of the line of anastomosis but this does not explain all of the cases. If it is accepted that the progressive element of coarctation is due to deposition of fibrin and platelets on an abnormal area of intima, any failure to remove the abnormal area completely would pre- 
dispose to recurrence even if there were no residual stenosis. If there were also irregularities of blood flow due to technical imperfections in the anastomosis the effect of any remaining intimal deformities would be enhanced, and the process of laminated intimal thickening could begin again.

We thank Professor W A J Crane, Mr G H Smith, Mr A C Welsford, Mr C A Lambourne, Mr M Eaton, the Department of Medical Photography, Northern General Hospital, and Miss J Mitchell.

\section{References}

Clagett, O T, Kirklin, J W, and Edwards, J E (1954). Anatomic variations and pathologic changes in coarctation of the aorta. Surgery, Gynecology and Obstetrics, 98, 103-114.

Clark, J M, and Glagov, S (1976). Luminal surface of distended arteries by scanning electron microscopy: eliminating configurational and technical artefacts. British Journal of Experimental Pathology, 57, 129135.

Edwards, J E, Christensen, N A, Clagett, O T, and McDonald, J R (1948). Pathologic considerations in coarctation of the aorta. Proceedings of the Staff Meetings Mayo Clinic, 23, 324-332.

Hutchins, G M (1971). Coarctation of the aorta explained as a branch point of the ductus arteriosus. American Journal of Pathology, 63, 203-209.

Talner, N S, and Berman, M A (1975). Postnatal development of obstruction in coarctation of the aorta; role of ductus arteriosus. Pediatrics, 56, 562569.

Wielenga, G, and Dankmeijer, J (1968). Coarctation of the aorta. Journal of Pathology and Bacteriology, 95, 265-274.

Requests for reprints to: Dr A Kennedy, Department of Histopathology, Northern General Hospital, Herries Road, Sheffield S5 7AU. 